\title{
Digital microfluidics with magnetically actuated floating liquid marble
}

\author{
Mei Kum Khaw, ${ }^{1}$ Chin Hong Ooi, ${ }^{1}$ Faisal Mohd-Yasin, ${ }^{1}$ Raja Vadivelu, ${ }^{2}$ James St \\ $\mathrm{John}^{2}$ and Nam-Trung Nguyen ${ }^{1 *}$
}

Received 00th February 2016 Accepted 00th February 2016

${ }^{1}$ Queensland Micro- and Nanotechnology Centre, Nathan campus, Griffith University, 170 Kessels Road QLD 4111, Australia. *E-mail: nam-trung.nguyen@griffith.edu.au ${ }^{2}$ Eskitis Institute for Drug Discovery, Griffith University, Brisbane, QLD 4111, Australia

www.rsc.org/softmatter

\begin{abstract}
Controlled actuation of a floating liquid marble, a liquid droplet coated with hydrophobic particles floating on another liquid surface, is a potential digital microfluidics platform for the transportation of aqueous solution with minimal volume loss. Compared to microchannelbased microfluidics, the lack of confinement in the discrete droplet allows for more versatile and diverse applications. This paper reports our recent investigation on the magnetic actuation of floating liquid marbles filled with magnetic particles. The magnetic force and the frictional force acting on the floating liquid marble determine the horizontal movement of the marble. We varied the magnetic flux density, flux density gradient, concentration of magnetic particles and the speed of the marble to elucidate the relationship between the acting forces. We subsequently determined the suitable operating conditions for the actuation and also derived the scaling laws for the parameters. Controlled manipulation of the floating liquid marble has the immediate application for growing three-dimensional cell cultures as floating liquid marbles enable cells within a droplet to freely interact to produce cell spheroids. Effective mixing inside the liquid marble is essential for the quality and reproducibility of the cell culture.
\end{abstract}

\section{Introduction}

A liquid marble is typically an aqueous solution encapsulated by a coating of hydrophobic powder. The powder is attracted to the liquid-air interface and forms a coating that isolates the liquid in a marble from the external surface it is resting on by an air layer. ${ }^{1,2}$ The surface can be a solid surface or the free surface of another aqueous solution. On a solid surface, a liquid marble can roll or slide around without wetting the surface. On an aqueous free surface, the liquid marble floats and slides with minimum friction.

The non-wetting feature of liquid marbles allows the transportation of liquid without volume loss and energy loss due to the low friction with the platform surface. A recent review by Ooi et al. ${ }^{3}$ demonstrated that a liquid marble can be actuated through electrostatic force, magnetic force, gravitational force, pressure gradient and chemical schemes. Therefore, the manipulation of liquid marbles is potentially useful for digital microfluidics. In comparison to microchannel-based microfluidics, manipulation of discrete droplets without being confined in a closed channel allows for versatile applications.

A liquid marble also has a lower evaporation rate as compared to a non-coated free droplet. Although the gas exchange within the liquid marble and surrounding environment cannot be avoided, the volume of a liquid marble can be maintained by changing the humidity and temperature of its surrounding. This characteristic makes liquid marbles suitable to serve as a cell culture platform, ${ }^{4,5}$ as a liquid marble can maintain its volume for several days. Vadivelu et al. ${ }^{5}$ used a floating liquid marble as a bioreactor for growing threedimensional cell spheroids. The floating liquid marble allows the cells to freely associate and interact to produce uniform sized spheroids. Efficient mixing inside a liquid marble is crucial for the effectiveness of the bioreactor. The quality of the spheroids depends on the internal mixing process, which in turn depends on the motion of the marble over the surface of the supporting liquid. Moving the marble around would induce internal flow and mixing. Ooi et al. ${ }^{6}$ reported the kinematics and dynamics of a floating self-propelling liquid marble containing ethanol. The self-driven movement is 
caused by the Marangoni solutocapillary effect, which is difficult to control and depends on the availability of ethanol acting as the fuel inside the marble. Our motivation for the present work is the development of a controllable magnetic actuation scheme for the floating liquid marble leading to a practical digital microfluidics platform. Liquid from the liquid marble loaded with magnetic particles can be extracted or added ${ }^{7}$ which enables the liquid marble to be used in micro reactor and micro mixer.

Recent works already described the kinematics and dynamics of magnetically actuated sessile droplet on a solid surface ${ }^{8-11}$. Nguyen et al. ${ }^{11}$ investigated the sliding motion of sessile water-based nonreactive ferrofluid droplets on hydrophobic planar surfaces using a moving external magnet. This work provided the critical velocities of the actuated droplets with different the magnetic flux density induced by different permanent magnets. Further work reported by Koh et al. ${ }^{10}$ demonstrated the use of magnetically actuated ferrofluid droplets as actuators for the key functions in digital microfluidics such as merging and mixing of other non-magnetic liquid droplets.

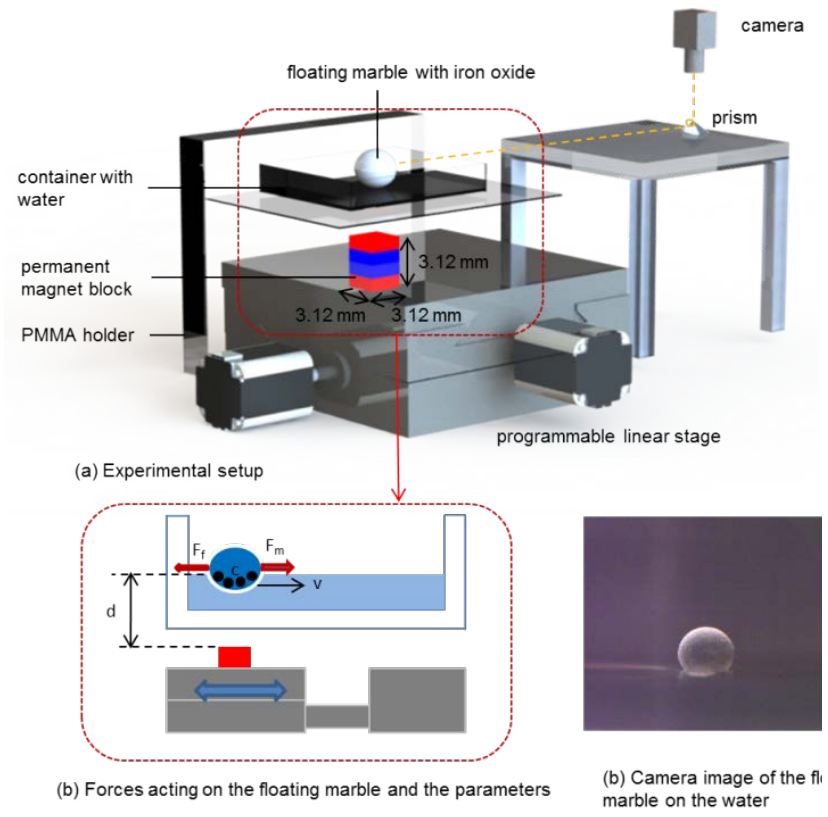

Figure 1: (a) The experimental setup; (b) Forces acting on the floating marble $\left(F_{\mathrm{m}}\right.$ represents the magnetic force and $F_{\mathrm{f}}$ represents the friction force). The parameters of the experiments are the distance between the floating marble from the magnet $d$, the concentration of the iron oxide particles $c$, and the velocity of the permanent magnet $v$; (c) A typical image of the floating marble on the water surface captured with the setup.

The drawback of this work was that the whole droplet should be magnetic, for example using ferrofluid, such that the magnetic force can overcome the friction with the solid surface. Ferrofluid is not compatible with biological applications such as cell culture. Actuation with a small amount of larger magnetic particles in the droplet would not work because the small magnetic force can not overcome the friction force or would be too large so that the particle is removed from the droplet. The solution for reducing the friction is to float the liquid marble on a liquid surface and then drag it with a minute amount of magnetic particles and an external magnet.

The objective of our present work is to study the sliding motion of a magnetically actuated floating marble. We experimentally evaluate the parameters that affect the motion of a floating liquid marble in terms of displacement and velocity such as the volume concentration of magnetic particles, the speed and the flux density of the driving permanent magnet. With the data collected, we can then determine the suitable operating conditions for the actuation as well as the critical points where the liquid marble no longer follows the permanent magnet. The scaling relationship of the operation parameters can be compared with the counterparts of a sessile droplet moving on a hydrophobic surface.

\section{Materials and Methods}

\section{Preparation of a floating marble for magnetic actuation}

The marble liquid is formed by mixing deionised water with iron oxide powder (Sigma-Aldrich, Iron II/III oxide). Magnetite particles are spherical with a diameter size of less than $5 \mu \mathrm{m}$ and a density of approximately 4.8 to $5.1 \mathrm{~g} / \mathrm{mL}$ at room temperature. The iron oxide was accurately weighed using a high precision analytical balance (Radwag, USA) with a resolution of $0.1 \mathrm{mg}$. For instance, a volume of $4 \mathrm{ml}$ deionized water was mixed with $12 \mathrm{mg}$ of iron oxide to generate a concentration of $0.003 \mathrm{~g} / \mathrm{ml}$ of magnetic particles. A microliter droplet of the magnetic solution was dispensed into a bowl filled with polytetrafluoroethylene (Teflon) powder and rolled into a liquid marble. The Teflon powder (Sigma Aldrich) has a particle size of $1 \mu \mathrm{m}$ and a density of approximately $2200 \mathrm{~kg} / \mathrm{m}^{3}$. A micropipette (Genex-Beta, UK) was used to accurately dispense $0.5 \mu \mathrm{L}$ to $10 \mu \mathrm{L}$ of the liquid. The liquid marble was continuously rolled until completely coated. The liquid marble was then picked up with a stainless steel spoon and further rolled around to remove the excess powder. The uniformly coated liquid marble was then carefully transferred to a polystyrene container which was initially filled with deionised water as its carrier liquid. The liquid marble floated on the surface and can be actuated by a moving an external permanent magnet.

\section{Experimental setup}

Our experimental platform consists of a holder made of poly methyl methacrylate (PMMA), a container made of polystyrene, a permanent magnet and a programmable $x-y$ stage, Figure 1. The holder is made of 4.5-mm thick PMMA plates and has a slit to hold a 1.5-mm thick PMMA plate for positioning the $10 \mathrm{~cm} \times 10 \mathrm{~cm}$ polystyrene container. The water levels to be filled were marked with a permanent marker on the polystyrene container. A cubic permanent magnet (Neodymium, $3.12 \mathrm{~mm} \times 3.12 \mathrm{~mm} \times 3.12 \mathrm{~mm}$ ) was fixed on a programmable $x-y$ stage. The permanent magnet was placed below the container as a tool to move the floating marble. This magnet was positioned at distances of $6 \mathrm{~mm}, 8 \mathrm{~mm}, 10 \mathrm{~mm}$, $12 \mathrm{~mm}$ and $14 \mathrm{~mm}$ from the water level markings. We used a venier calliper to accurately adjust the distance, taking into account the 
combined thickness of $2.5 \mathrm{~mm}$ of the PMMA plate and the polystyrene container.

The $x-y$ stage was a motorized linear actuator (Zaber Technologies T-LS28M) with built-in controllers. The actuator has a travel range of $28 \mathrm{~mm}$, a maximum speed of $6.5 \mathrm{~mm} / \mathrm{s}$ and a minimum speed of $0.93 \mu \mathrm{m} / \mathrm{s}$. The linear motion of the actuator was controlled by the Zaber Console software. Codes were programmed to define the velocity of the actuator. The actuator was linked to the PC through a RS-232 port. The actuator allowed a programmable and smooth motion of the permanent magnet in both directions.

The experimental setup facilitates video capturing of the moving liquid marble. The video of the floating marble were recorded and further processed to examine the motion. For this purpose, a $45^{\circ}$ prism mirror was placed under the camera to capture the side view of the floating marble without blocking the x-y stage and the container. The floating marble was backlit with a light source to create a large contrast between the marble and its surrounding. The images were captured by an USB camera (Edmund Optics Inc, Singapore) with an

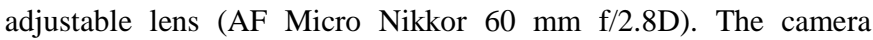
software (uEye cockpit, IDS, Germany) was set to record the motion with 30 frames per second (fps) in a single movie file. In the subsequent image-processing step, the frames were separated and evaluated individually using ImageJ (National Institutes of Health, USA) and a custom-written program in Matlab (Mathworks, USA), to obtain an objective assessment of the motion (displacement, velocity and acceleration) of the floating marble.

In the experiments, deionised water was filled into the container until it reached the first marked level, representing $6 \mathrm{~mm}$ distance from the magnet. A $3 \mu \mathrm{L}$ magnetised liquid marble was carefully placed into the container. The floating marble automatically aligned itself to the centre of the permanent magnet. The magnet was programmed to move back and forth with a pre-set amplitude of $4.96 \mathrm{~mm}$ and 9 speeds ranging from $0.465 \mathrm{~mm} / \mathrm{s}$ to $4.19 \mathrm{~mm} / \mathrm{s}$. Next, $0.2 \mathrm{ml}$ of deionised water was drawn with a syringe and added into the container corresponding to an increase of $2 \mathrm{~mm}$ in the water level. The experiment was repeated for the different water levels. The motions of the floating marble are recorded and analysed for each water level or marble-magnet distance and its corresponding speed. The Matlab program then processed the video and measured and tracked the centroid of the floating marble as it moved back and forth with the permanent magnet.

\section{Results and Discussion}

\section{Forces acting on the magnetic floating marble}

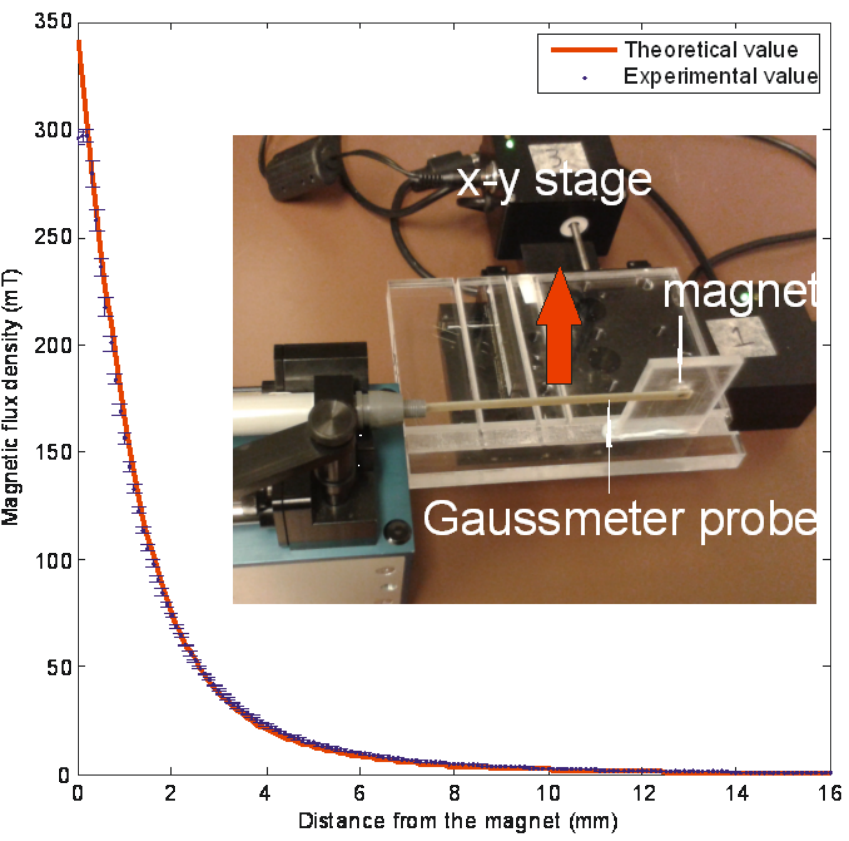

Figure 2: Magnetic flux density versus the distance to the permanent magnet. Points with error bars are measurement data, line is the plot of Eq. (2). Inset: Setup of the measurement

When the liquid marble is transferred to the water container, it floats on the water surface. The vertical component of the magnetic force and the weight of the liquid marble balance the upward acting buoyancy force and surface tension force. The vertical non-magnetic forces were analysed and discussed in our recent work. ${ }^{11}$ For the actuation scheme of the present work only horizontal forces are considered.

Assuming the surrounding medium is diamagnetic, the driving force acting on a floating marble within the field of the permanent magnet) is given by ${ }^{12,13}$

$$
F_{\mathrm{m}}=\frac{V \chi}{\mu_{o}} B \frac{\mathrm{d} B}{\mathrm{~d} x}
$$

where $F_{\mathrm{m}}$ is the magnetic force, $V$ is the volume of the iron oxide, $\chi$ is the magnetic susceptibility of the floating marble, $\mu_{0}$ is the permeability of vacuum, $B$ is the magnetic flux density. Since the marble volume in our experiments is fixed, the magnetic force scales with the flux density and the flux density gradient of the magnetic field and the concentration of iron oxide inside the marble:

$$
F_{\mathrm{m}} \propto V B \frac{\mathrm{d} B}{\mathrm{~d} x}
$$

Thus, in the subsequent experiments, varying the concentration of iron oxide and the distance between the marble and the permanent magnet allows the adjustment of the magnetic force. 
(a) $\mathrm{d}=6 \mathrm{~mm}$
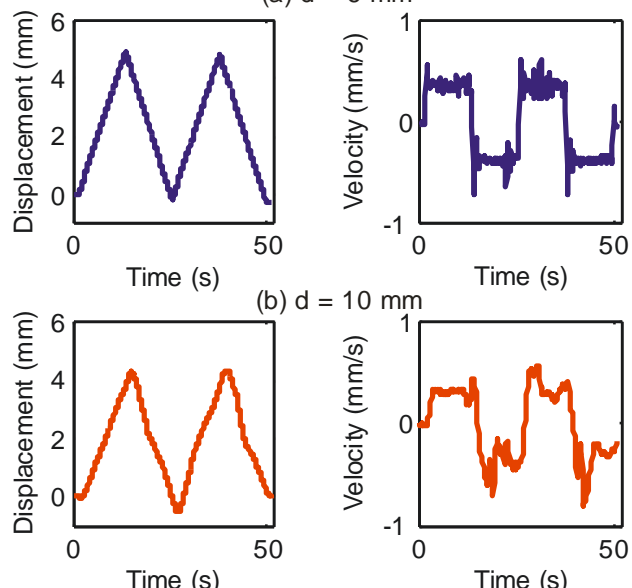

(b) $\mathrm{d}=10 \mathrm{~mm}$

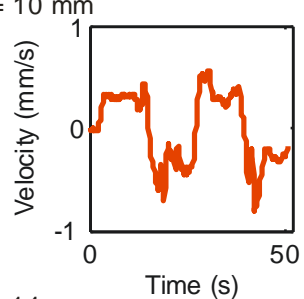

(c) $\mathrm{d}=14 \mathrm{~mm}$
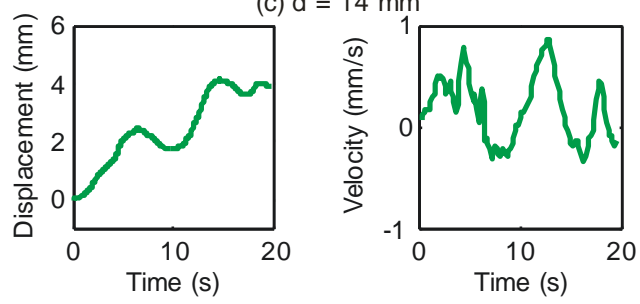

Figure 3: Measured displacement and velocity of a $3-\mu \mathrm{L}$ floating liquid marble with an iron oxide concentration of $0.003 \mathrm{~g} / \mathrm{ml}$ and a velocity of the permanent magnet of 0.465 $\mathrm{mm} / \mathrm{s}$ with increasing distance from the magnet; (a) $d=6 \mathrm{~mm}$ ( $B=10.1 \mathrm{mT})$; (b) $d=10 \mathrm{~mm}(B=3.09 \mathrm{mT})$; and (c) $d=14 \mathrm{~mm}$ $(B=1.35 \mathrm{mT})$

The magnetic flux density of the magnet, $B$ was measured with a handheld commercial gaussmeter (GM07, measurement range from 0 to $3 \mathrm{~T}$, Hirst Magnetic Instruments Ltd, UK). Figure 2 shows the measured magnetic flux densities with increasing distance of the gaussmeter probe from the magnet. The magnet is mounted vertical onto a PMMA holder and placed on the $x-y$ stage. Initially, the probe touches the magnet to get the maximum reading. The $x-y$ stage is then programmed to move $0.1 \mathrm{~mm}$ away from the probe for each measurement. The measured result agreed well with the theoretical estimation of the magnetic field:

$$
\begin{aligned}
B=\frac{B_{\mathrm{r}}}{\pi}\left[\arctan \left(\frac{L W}{2 z \sqrt{4 d^{2}+L^{2}+W^{2}}}\right)\right. & \\
& \left.\quad-\arctan \left(\frac{L W}{2(D+d) \sqrt{4(D+d)^{2}+L^{2}+W^{2}}}\right)\right]
\end{aligned}
$$

where $B_{\mathrm{r}}$ is the remanence field, $L$ is the length, $W$ the width and $D$ the height of the magnet, $d$ is the distance from the magnet surface.

From the experimental data in Fig. 2 and equation (3), the relationship between flux density $B$ and the distance $d$ can be simplified to the scaling relationship $B \propto \frac{1}{d}$. Thus, the distance of the floating marble from the permanent magnet $d$ as well as the (a) $c=0.005 \mathrm{~g} / \mathrm{ml}$
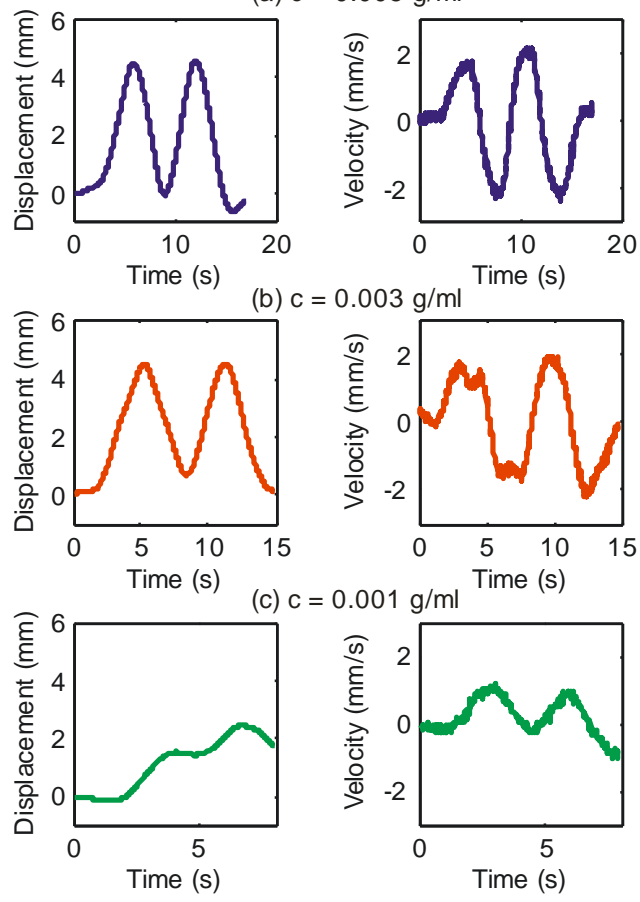

Figure 4: Measured displacement and velocity of a $3-\mu \mathrm{L}$ floating marble at a distance of $10 \mathrm{~mm}$ from the permanent magnet and a velocity of the permanent magnet of $1.86 \mathrm{~mm} / \mathrm{s}$ with decreasing iron oxide concentration; (a) $c=0.005 \mathrm{~g} / \mathrm{ml}$; (b) $c=0.003 \mathrm{~g} / \mathrm{ml}$; and (c) $c=0.001 \mathrm{~g} / \mathrm{ml}$

concentration of iron oxide $c$ will affect the driving magnetic force of the marble. We hypothesise that if $d$ increases and $c$ decreases, the magnetic force becomes weaker. As result, the floating marble will start to lag behind the moving permanent magnet until reaching a limit and dislodge.

The force dragging the floating marble back is the friction force, $F_{\mathrm{f}}$ and is given by ${ }^{12}$

$$
F_{\mathrm{f}} \propto A \mu v
$$

where $A$ is the contact area between the marble and the carrying liquid, $\mu$ is the viscosity of the carrying liquid and $v$ is the velocity of the floating marble and the magnet. As the volume of floating marble and the medium is fixed, we assumed that the contact area remains constant throughout the experiment and the scaling relationship for $F_{\mathrm{f}}$ is

$$
F_{\mathrm{f}} \propto v
$$

In reality, the contact area between the marble and the carrying liquid could increase with a larger magnetic force. To simplify the analysis, and as mentioned previously, we ignore the effect of the vertical component of the magnetic force that affects the contact area. The relationship (6) indicates that increasing velocity of the magnet will increase friction force $F_{\mathrm{f}}$ causing the floating marble to lag behind the moving permanent magnet and eventually to dislodge. 

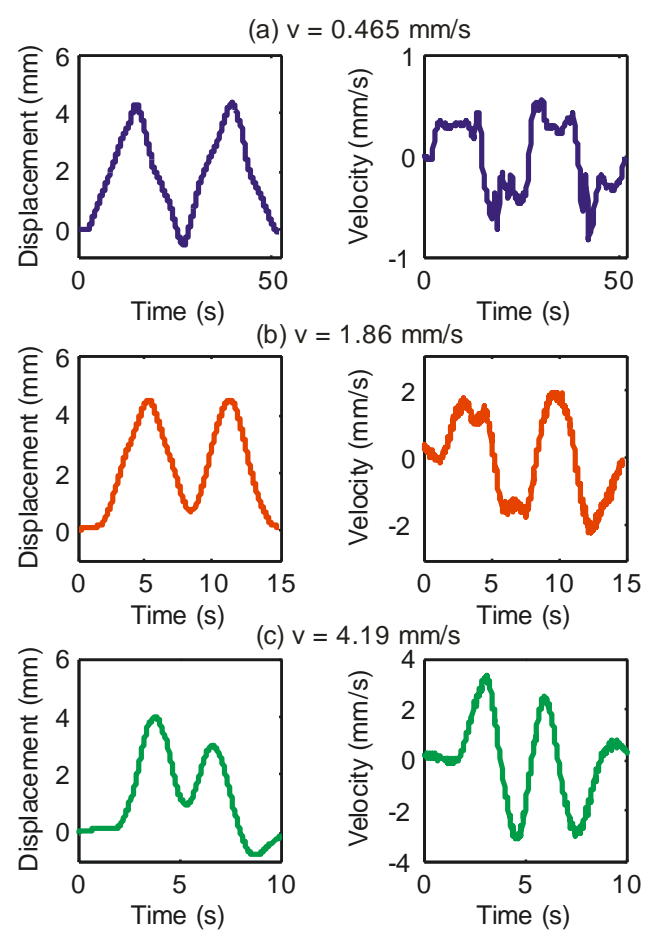

Figure 5: Measured displacement and velocity of a $3-\mu \mathrm{L}$ floating marble at a distance of $10 \mathrm{~mm}$ from the permanent magnet and with an iron oxide concentration of $0.003 \mathrm{~g} / \mathrm{ml}$ with increasing velocity of the magnet (a) $v=0.465 \mathrm{~mm} / \mathrm{s}$, (b) $v=1.86 \mathrm{~mm} / \mathrm{s}$ and (c) $v=4.19 \mathrm{~mm} / \mathrm{s}$

\section{Sliding of the floating liquid marble}

Because the steady motion of the floating marble results from the balance between the horizontal component of the magnetic force and the friction force, both magnetic force and friction force were varied in our experiment to determine the operation map. We investigate the movement of the floating marble in terms of displacement and velocity with the variation of each parameter. According to (2), the magnetic force can be tuned by adjusting the flux density $B$ and the flux density gradient $\mathrm{d} B / \mathrm{d} x$ through the distance $d$ between the magnet and the marble. The magnetic force will decrease with the increasing distance. Another way to vary the magnetic force is to change the volume $V$ of the iron oxide particles. A higher concentration will result in a larger volume of iron oxide for actuation. According to equation (5), the friction force is adjusted with the velocity $v$ of the permanent magnet. The higher the velocity, the larger is the friction force.

Three parameters were varied in our experiment: five different distances of floating marble from the magnet $d$ ranging from $6 \mathrm{~mm}$ to $14 \mathrm{~mm}$, three concentrations of iron oxide $c$ ranging from 0.001 $\mathrm{g} / \mathrm{ml}$ to $0.005 \mathrm{~g} / \mathrm{ml}$ and nine magnet velocities $v$ ranging from 0.465 $\mathrm{mm} / \mathrm{s}$ to $4.19 \mathrm{~mm} / \mathrm{s}$.

If the permanent magnet is slow and close to the marble, the resulting strong magnetic force causes the floating marble to slide and to follow the magnet with the same velocity. For the potential use of this system as a digital microfluidics system, this is the preferred operating condition. Figure 3(a) shows a smooth marble movement from stationary to a distance of of $4.96 \mathrm{~mm}$ before turning back to the other direction, as programmed for the magnet. Figures 3(b) and (c) show the behaviour of the marble with increasing distance from the magnet, while the iron oxide concentration and the velocity were fixed at $0.003 \mathrm{~g} / \mathrm{ml}$ and $0.465 \mathrm{~mm} / \mathrm{s}$, respectively. At $d=10 \mathrm{~mm}$, the marble starts to lag behind the magnet. A further distance of $d=14 \mathrm{~mm}$, the marble dislodged from the movement of the permanent magnet. Although the initial driving force is big enough to induce a momentum to the marble, however the friction force is dominant and the marble cannot keep up with the magnet.

Figure 4 shows the representative behaviour of the marble with varying concentration of the iron oxide. The distance from the magnet was chosen as $d=10 \mathrm{~mm}$, and velocity was fixed at $v=1.86 \mathrm{~mm} / \mathrm{s}$. The marble slides along with the magnet at the concentrations of $0.003 \mathrm{~g} / \mathrm{ml}$ and $0.005 \mathrm{~g} / \mathrm{ml}$. However, at the concentration of $0.001 \mathrm{~g} / \mathrm{ml}$, the floating marble no longer follows the moving magnet. (a) $c=0.003 \mathrm{~g} / \mathrm{ml}$

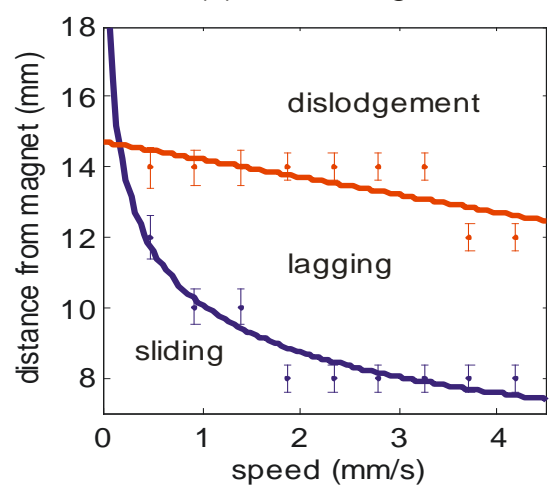

(b) $c=0.005 \mathrm{~g} / \mathrm{ml}$

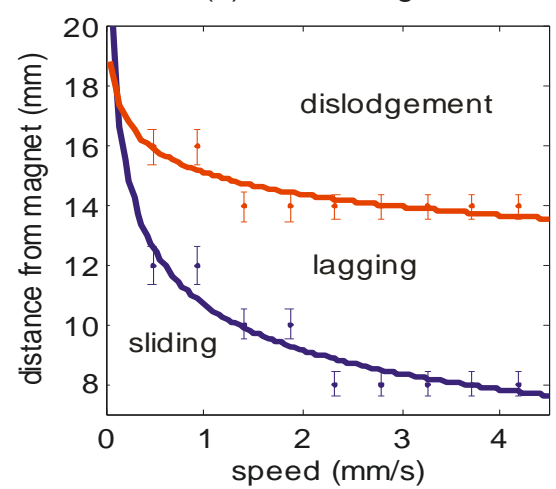

(c) $\mathrm{d}=10 \mathrm{~mm}$

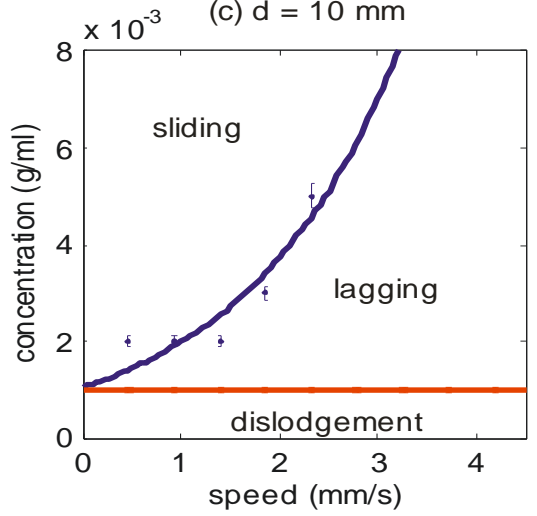

Figure 6: Operating map with the three regimes sliding, lagging and dislodgement. (a) Distance from magnet and magnet speed space at $c=0.003 \mathrm{~g} / \mathrm{ml}$; (b) Distance from magnet and magnet speed at $c=0.005 \mathrm{~g} / \mathrm{ml}$; and (c) Iron oxide concentration and speed space. 


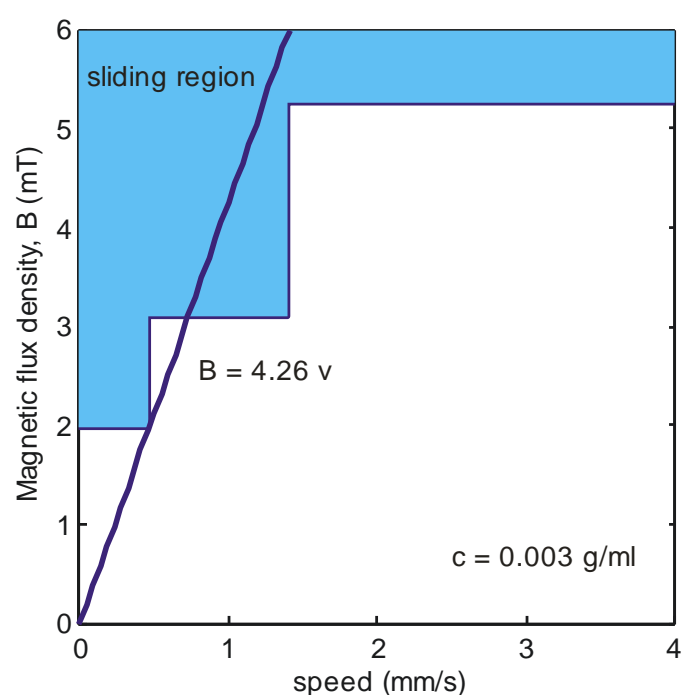

(a)

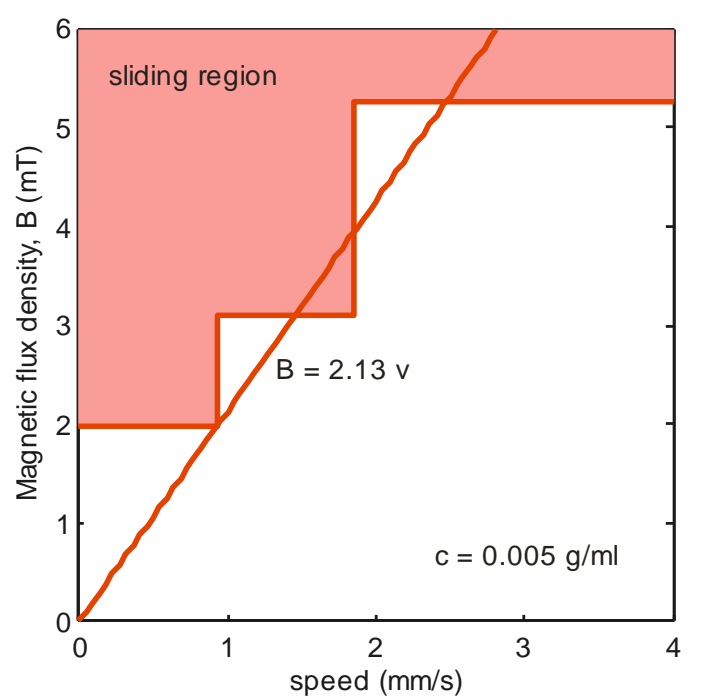

(b)

Figure 7: Operating region of the floating marble in our experiments. The blue-shaded area (a) is the sliding region with the floating marble with a concentration of $0.003 \mathrm{~g} / \mathrm{ml}$ and the red-shaded area (b) is the sliding region with the floating marble with a concentration of $0.005 \mathrm{~g} / \mathrm{ml}$.

Figure 5 shows the representative behaviour of the marble with increasing velocity. At a high velocity, the friction force becomes dominant and the marble tends to lag behind. The marble slides with the magnet with velocity ranging from $0.465 \mathrm{~mm} / \mathrm{s}$ to $1.86 \mathrm{~mm} / \mathrm{s}$. Beyond that, the marble lags behind until it starts to dislodge at $4.46 \mathrm{~mm} / \mathrm{s}$.

As a summary, Figure 6 shows the operating maps of the floating marble when the $d, c$ and $v$ are varied. Three zones were identified: sliding, lagging and dislodgment. When $F_{\mathrm{m}} \approx F_{\mathrm{f}}$, the marble slides following the magnet to the preprogramed distance of $4.96 \mathrm{~mm}$ with the same velocity as that of the magnet. When $F_{\mathrm{m}}<F_{\mathrm{f}}$, the marble lags behind and if the maximum velocity is reached, it will dislodge from the influence of the magnet. Figures 6(a) and (b) show the operating map of two different concentrations of iron oxide. A higher concentration shows the better control of the movement when the distance of the marble from magnet and speed increased. Figure 6(c) shows the operation map with concentration as one parameter, a concentration of $0.001 \mathrm{~g} / \mathrm{ml}$ and below is not strong enough to let the marble follow the magnet.

\section{Scaling Analysis and Operating Regions}

At the lowest magnetic flux density $B_{\min }$, the floating marble follows the magnet until the maximum velocity is reached. To simplify the equation for scaling analysis, the force balance in the sliding operating region is

$$
\begin{aligned}
& F_{\mathrm{m}}=F_{\mathrm{f}} \\
& \alpha B_{\text {min }}=\beta v_{\text {max }} \\
& B_{\text {min }}=\frac{\beta}{\alpha} v_{\text {max }}
\end{aligned}
$$

where $\alpha$ and $\beta$ are simply scaling factor. Figure 7 replots the data of Figure 6(a) and (b) with the actual flux density converted from the distance using Fig. 2. The shaded areas represent the experimentally obtained sliding region of the marble. In this region, the driving magnetic force overcomes the frictional force which allows the marble to follow the permanent magnet where $F_{\mathrm{m}} \geq F_{\mathrm{f}}$.

A scaling relationship of (8) is fitted to the experimental data of Fig. 7. To get the slope $\beta / \alpha$, we chose the critical condition for the sliding regime for both of the concentrations. The minimum magnetic flux density of $1.98 \mathrm{mT}$ and maximum velocity of 0.465 $\mathrm{mm} / \mathrm{s}$ were chosen for the concentration of $0.003 \mathrm{~g} / \mathrm{ml}$. The minimum magnetic flux density of $1.98 \mathrm{mT}$ and maximum velocity of $0.930 \mathrm{~mm} / \mathrm{s}$ were chosen for the $0.005 \mathrm{~g} / \mathrm{ml}$ concentration. The slopes are $4.26 \mathrm{Ts} / \mathrm{m}$ and $2.13 \mathrm{Ts} / \mathrm{m}$ respectively. Referring back to equation (2) and taking into account the concentration of iron oxide is directly proportional to its volume, $\alpha$ represents the term $\frac{c \chi}{\mu_{o}} \cdot \frac{\mathrm{d} B}{\mathrm{~d} x}$. Whilst $\frac{\chi}{\mu_{o}}$ remain the same for both cases, we find the product of the proportional factor and the concentration. The data in Figure 7(a) leads to $B=(4.26 \times 0.003) v=0.013 v$, while the data in Figure $7(\mathrm{~b})$ leads to $B=(2.13 \times 0.005) v=0.011 v$. The slight difference may be attributed to the term $\mathrm{d} B / \mathrm{d} x$. Thus, discounting the concentration of iron oxide, the data of Figs. 7(a) and 7(b) fit relatively well.

\section{Conclusion}

This paper investigates experimentally the sliding motion of a magnetically actuated floating liquid marble. Using a permanent magnet, the movement of a floating liquid marble consisting of iron oxide is able to be controlled. While the shape and size of the permanent magnet were fixed, the concentration of iron oxide in the marble and the distance of the marble from the permanent magnet were varied. The key to a controlled movement is to supply adequate actuation force to 
oppose its frictional force, which is contributed by the velocity of the pulling permanent magnet. In general, the suitable sliding condition is a low speed of the permanent magnet, a high magnetic flux density and a high concentration of magnetic particles in the floating liquid marble. The critical magnetic parameters and velocities derived from the scaling analysis fit well to the experimental data describing the sliding region. The work reported he lays the foundation for the precise control of a floating liquid marble and consequently its internal flow field and chaotic mixing. Effective mixing inside the liquid marble has the immediate application for growing three-dimensional cell cultures as it enable cells within the marble to freely interact to produce cell spheroids.

\section{Acknowledgements}

We acknowledge Queensland Micro- and Nanotechnology Centre for providing the facilities to make the experiments possible. This work was performed in part at the Queensland node of the Australian National Fabrication Facility, a company established under the National Collaborative Research Infrastructure Strategy to provide nano- and microfabrication facilities for Australia’s researchers.

\section{References}

1. P. Aussillous and D. Quéré, Nature, 2001, 411, 924-927.

2. E. Bormashenko, Y. Bormashenko, A. Musin and Z. Barkay, ChemPhysChem, 2009, 10, 654-656.

3. C. H. Ooi and N.-T. Nguyen, Microfluidics and Nanofluidics, 2015, 1-13.

4. J. Tian, N. Fu, X. D. Chen and W. Shen, Colloids and Surfaces B: Biointerfaces, 2013, 106, 187-190.

5. R. K. Vadivelu, C. H. Ooi, R.-Q. Yao, J. T. Velasquez, E. Pastrana, J. Diaz-Nido, F. Lim, J. A. Ekberg, N.-T. Nguyen and J. A. St John, Scientific reports, 2015, 5.

6. C. H. Ooi, A. van Nguyen, G. M. Evans, O. Gendelman, E. Bormashenko and N.-T. Nguyen, RSC Advances, 2015, 5, 101006-101012.

7. Y. Zhao, J. Fang, H. Wang, X. Wang and T. Lin, Advanced materials, 2010, 22, 707-710.

8. L.-H. Lu, K. S. Ryu and C. Liu, Microelectromechanical Systems, Journal of, 2002, 11, 462-469.

9. Z. Long, A. M. Shetty, M. J. Solomon and R. G. Larson, Lab on a Chip, 2009, 9, 1567-1575.

10. W. H. Koh, K. S. Lok and N.-T. Nguyen, Journal of Fluids Engineering, 2013, 135, 021302.

11. C.H. Ooi, C. Plackowski, A.V. Nguyen, R.K. Vadivelu, J. A. St John, D.V. Dao, N.-T. Nguyen, Floating mechanism of a small liquid marble, Scientific Reports, 2016, Vol. 6, pp. 21777.

12. N.-T. Nguyen, G. Zhu, Y.-C. Chua, V.-N. Phan and S.-H. Tan, Langmuir, 2010, 26, 12553-12559.

13. S. S. Shevkoplyas, A. C. Siegel, R. M. Westervelt, M. G. Prentiss and G. M. Whitesides, Lab on a chip, 2007, 7, 1294-1302. 